\title{
UNEXPLAINED FEVER IN HEART FAILURE
}

\author{
By ALFRED E. COHN AND J. MURRAY STEELE \\ (From the Hospital of the Rockefeller Institute for Medical Research, \\ New York City)
}

(Received for publication July 2, 1934)

\section{HISTORICAL INTRODUCTION}

Very early the occurrence of fever during disease was commented upon by Hippocrates as one of its most important symptoms but not until more than 2000 years later, in the early part of the seventeenth century, were quantitative measurements of the temperature of the body obtained. Sanctorius employed a thermometer of his own making for this purpose. Still another century elapsed, however, before the significance of such measurements began to be recognized. Boerhaave occasionally used a thermometer as a practical aid in diagnosis but left for his pupils Van Swieten and De Haen, the first professor of clinical medicine at Vienna, the chance to record the useful, if naive, observation that external temperature was more easily recognized by the thermometer but that internal temperature was still best described by symptom and sign. It is of considerable interest that "internal heat" or temperature was described by the iatro-physicians in terms of disturbance of circulation and its presence was decided upon by the frequency and quality of the pulse. One view accounted for the production of heat mechanically by the friction of blood moving in the vessels but was opposed both by De Haen and John Hunter. When Lavoisier and Laplace announced (1780) that the source of animal heat was combustion of organic substances in the body, the theory of mechanical production of heat was quickly discarded in favor of one chemical in nature. The belief of Lavoisier that heat was generated in the lungs during oxygenation of the blood was resisted by Brodie (1811) who looked for its source in the nervous system. There followed many observations on the relation of the nervous system to the temperature of the body by Chossat (1820), Edwards (1824), Home (1825), and later Claude Bernard (1852). These researches succeeded, not in establishing the location of the source of heat, but in leading to an understanding of the extraordinary degree of control which the nervous system exerts over the temperature of the body.

While these physiological studies of the regulation of temperature were being carried out, records of the temperature of the body were being obtained by practicing physicians. In France, Piorry, Gierse, Roger; in England, Martine, Currie and Davy; in Germany, Zimmermann, Traube and von Bärensprung made numerous observations in health and in disease. Attempts to obtain more than one or two records of temperature during the course of an illness were rare although it is true that Davy, in mapping out physiological variations, and Traube, in attempting to follow closely the influence of taking digitalis on the temperature of the body and the course of events on critical days in febrile illnesses, occasionally made frequent measurements during the twenty-four hours. 
Wunderlich (1) was the first, however, to recognize clearly and to demonstrate the diagnostic and prognostic importance of a series of measurements taken throughout the course of a disease. He recorded the temperature of patients usually as often as every four hours and constructed charts showing its variations. He published clear descriptions regarding changes in the temperature of the body during many febrile diseases. The temperature charts of today are an expression of the universal recognition of the value of this procedure and of the technical convenience afforded by the use of Sir Clifford Allbutt's recording clinical thermometer.

Wunderlich's method of taking measurements frequently enough to obtain curves of the course of the rectal temperature characteristic of different diseases was imitated rapidly in England by Ogle, Aitken, and Gibson. Jurgenson and Wunderlich's pupils established it in Germany. The period in the nineteenth century that witnessed the introduction of thermometry into clinical medicine was characterized also by significant advances in knowledge concerning infectious diseases. The form of the record of temperature of the body became one of the principal means of characterizing various febrile affections and of recognizing complications of these diseases. The close association of infection with fever was soon established and stressed so energetically that at present physicians find it difficult to dissociate the occurrence of fever from its usual association with an infectious ailment.

It is well recognized, however, that many conditions other than infectious disease may be associated with elevation of the body's temperature, such as injury to certain parts of the brain, extravasation of blood into tissue spaces, infarcts, the state of hyperthyroidism and of sunstroke in which there is no reason to suspect the presence of infection.

When fever is observed in patients suffering from heart failure its nature has usually been ascribed to some sort of cryptic infection. And since patients with heart failure frequently suffer simultaneously from infection, the two conditions do in fact often coexist. Sometimes neither infection nor a non-infectious condition recognized as commonly associated with fever can be demonstrated. Under these circumstances physicians have generally assumed that infection is present but that the methods available are insufficient to disclose the nature or the location of the process. Because of the similarity of the physical signs of pulmonary infections and chronic passive congestion of the lungs, the lungs are usually regarded as infected though the possibility is recognized that infectious foci may exist in other organs as in the tonsils, the kidneys, or indeed, according to Romberg (2), in the heart itself.

Observations of the behavior of certain cardiac patients with fever have brought into question the wisdom of assuming in instances of unexplained fever the presence of an infectious process. For this reason the records of 368 cardiac patients coming under observation between 1914 and 1929 have been studied. Of 172 who presented symptoms or signs of heart failure, 153 exhibited, on two or more occasions, elevation of the rectal temperature to at least $100^{\circ} \mathrm{F}$. Usually the elevations were clearly associated with con- 
ditions generally recognized as accompanied by fever, but in 49 cases the occurrence of fever was without satisfactory explanation. In certain ones its development suggested an origin, at least in part, dependent upon heart failure itself.

Five cases are described to illustrate this conception:

Case I. G. M., Hospital Number 2098, male, aged 50 years, was admitted to hospital May 19, 1914, complaining of shortness of breath, weakness, and pain in the limbs.

His family history did not contribute to an understanding of his illness. His health had been excellent. Measles and pertussis in childhood, gonorrheal urethritis at the age of twenty and pain in the joints without swelling for five weeks at forty-three years of age had been his only illnesses. Two weeks after an attack of grippe $21 / 2$ months before admission, edema of the legs, pain in the back, shoulders and legs, dyspnea and weakness made their appearance. In retrospect he believed that he had been slightly short of breath for a year. $\mathrm{He}$ was an elderly, well-developed man, restless and slightly dyspneic. A mucopurulent post-nasal discharge was observed. The heart was enormously enlarged, the maximum transverse diameter of its shadow measuring $20.5 \mathrm{~cm}$. Auricular fibrillation was present; a systolic thrill was felt and systolic and diastolic murmurs were heard at the apex. The systolic blood pressure measured $189 \mathrm{~mm} . \mathrm{Hg}$ and the diastolic 100 . The lungs were clear. The liver extended only $1 \mathrm{~cm}$. below the costal margin. There was no edema of the legs and no cyanosis. The urine contained albumin and the sediment, a few red and white blood cells and numerous hyaline and finely granular casts. The Wassermann reaction of the blood was negative.

During the first two weeks he grew slowly worse; edema increased, the liver enlarged, râles were heard at the bases of the lungs, and the cardiac rate became accelerated. On June 3, digipuratum was given, followed by diuresis and disappearance of these signs. The administration of digipuratum was then omitted. During the next ten days the symptoms and signs gradually reappeared together with elevation of the rectal temperature. All symptoms were again relieved by the administration of the tincture of digitalis, begun July 1 (Fig. 1). The fever also subsided. During the presence of fever no obvious source of infection was found. The upper respiratory passages were normal and no signs of consolidation appeared in the lungs. The urine was clear. He was discharged July 17,1914 , fairly well and was instructed to continue to take the tincture of digitalis. He was readmitted on September 2, 1914. Periods during which digipuratum was given alternated with others free of it. When he was without digitalis edema and dyspnea increased, râles were heard in the lungs, the liver enlarged, the frequency of the heart beat increased, cyanosis made its appearance and the temperature of the body became elevated. Administration of the drug was followed by the disappearance of these signs and symptoms. Two of these attacks of failure, the first culminating on the 16th October, the second on the 19th November, 1914, were accompanied by the appearance of jaundice. The day after the first of these peaks dullness at the base of the right lung posteriorly and alteration of breath sounds were found. These signs persisted until November 28, and were apparently due to accumulations of fluid. On December 12, 1914, he was discharged free of the signs of heart failure. He was admitted again on May 24, 1915, in a state of extreme heart failure with Cheyne-Stokes breathing, jaundice and fever (Fig. 1, 


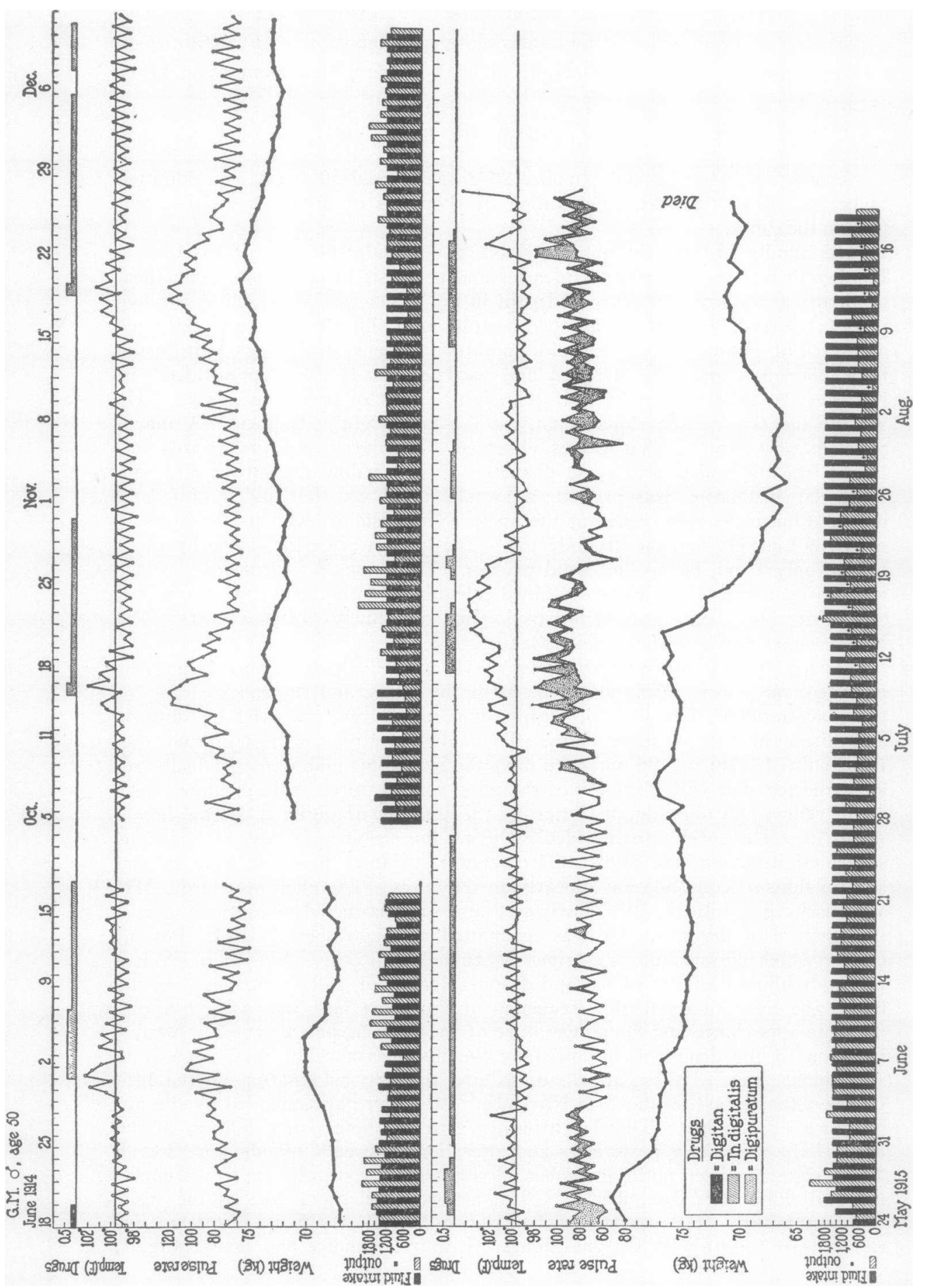

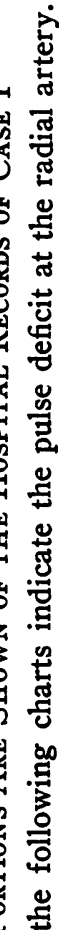

. 㟧

쿠

沓

:

.$\Xi$

ฮँ

Z

营

$\stackrel{\Xi}{E}$ 
lower half). Taking digitalis was followed by some improvement and disappearance of fever. The ground gained was rapidly lost when its administration was stopped because of the development of coupled rhythm. Renewed development of edema, increase in pulse rate, dyspnea and cyanosis and elevation of the temperature of the body recurred so promptly that diuretin was administered. Some decrease of the edema took place. As soon as the coupled rhythm disappeared, digipuratum was given again. The improvement which occurred was short lived however and on August 21, the patient died. Postmortem his heart was enlarged. There was chronic passive congestion of the liver, kidneys and lungs; right hydrothorax (200 cc.), ascites (300 cc.), and old fibrous pleural adhesions on both sides were found.

Case II. M. C., Hospital Number 4425, male, aged 33 years, a shipping clerk, was first admitted on December 1, 1921, complaining of heart trouble. The family history was of no significance. The past was free of any ailments including those of the "rheumatic group." $\mathrm{He}$ fell ill in June 1918, at age 29 , with cough, shortness of breath and occasional hemoptyses. A physician told him that he had heart trouble. He stopped work for six months, two weeks of which were spent in the Beth Israel Hospital. Later five similar attacks occurred during the last of which he was admitted to the Hospital of the Rockefeller Institute, two months after the onset of symptoms.

All the abnormal physical signs were referable to the cardiovascular system. A rapid precordial "undulation" was noted. No shocks or thrills were felt. The widest measurement of relative cardiac dullness extended $4.5 \mathrm{~cm}$. in the 4th interspace to the right and $11.5 \mathrm{~cm}$. in the 5th interspace to the left of the midline. The rhythm was rapid and altogether irregular (auricular fibrillation). The pulmonic second sound was louder than the aortic. No murmurs were heard. The systolic blood pressure measured $108 \mathrm{~mm}$. $\mathrm{Hg}$ and the diastolic 86. There was no enlargement of the heart nor was there an unusual contour in the $\mathrm{x}$-ray photograph. The radial pulse was of fair volume; the rate was 88 per minute while that of the apex was 132 . The edge of the liver was felt about $6.0 \mathrm{~cm}$. below the costal margin but was not tender. There was no edema of the extremities. The urine contained considerable albumin but no casts or red blood cells. Benedict's solution was faintly reduced in one of many examinations. The Wassermann reaction of the blood was negative.

After 3 days during which the apical rate varied between 130 and 190, with a pulse deficit of 50 to 100 per minute, and the rectal temperature ranged from 100 to $101.8^{\circ} \mathrm{F}$., dyspnea and cyanosis became so intense that digitan (Merck) 2 grams, was administered in the next succeeding 48 hours. The rapid cardiac rate, fever, and dyspnea subsided, cyanosis disappeared and in spite of the fact that no visible edema was present diuresis occurred with a loss of 9 kilograms in 8 days. Ten days later the administration of quinidine sulphate $1.2 \mathrm{gram}$ was followed by return of the normal rhythm. On January 25,1922 , free of any signs of heart failure he was discharged. He remained well without medication until June 1925, when auricular fibrillation recurred. $\mathrm{He}$ was given a large amount of digitalis to take, before being readmitted to observe the effect of administering quinidine. On June 22, 1925, 1.2 gram of quinidine was given, and on June 28, 1.6 gram without success in restoring normal rhythm. After taking 2.0 grams on June 29 , reversion to normal rhythm took place during the night (Fig. 2). Since then and until June, 1933, the rhythm of the heart has been normal. 
Case III. P. O., Hospital Number 3968, aged 53, male, was admitted on September 29, 1919, complaining since May, 1919, of palpitation and a sense of oppression in the chest. He had a chancre at the age of 23 treated with medicine by mouth for a year, and ten years later another penile lesion treated

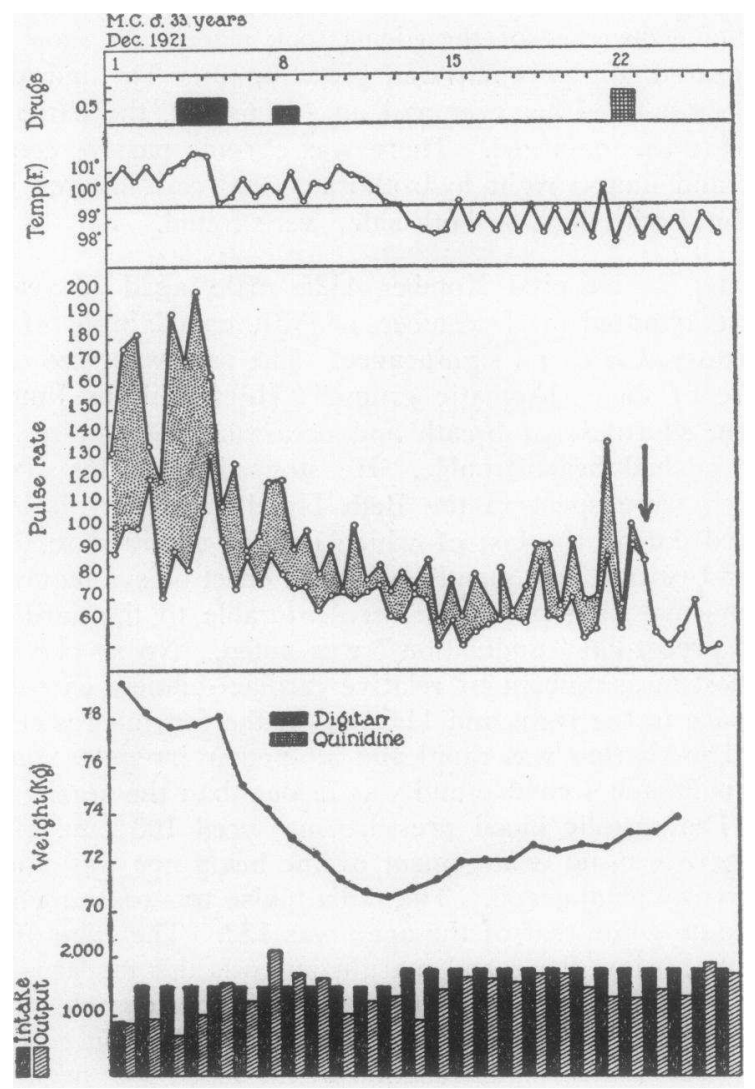

Fig. 2. The First Portion of the Record of Case II Is Exhibited

An elevation of temperature on December 21 accompanies increase of the cardiac rate after the injection of atropine sulphate intravenously.

by local fulguration. At the age of 46 years an attack of rheumatism (?) occurred with painful swollen joints but no fever. At age 52 a similar attack involved only the right knee. The palpitation and the smothering sensation in the chest which appeared May, 1919, were quickly followed by dyspnea on exertion and edema of the feet which resulted in his stopping work.

His general physique and nutrition were good. No dyspnea, orthopnea, cyanosis or edema was noted. Advanced dental caries and pyorrhea were present. The heart was enlarged, the sounds clear. There were no murmurs. The totally irregular rhythm was due to auricular fibrillation. The rate at the apex was 105, the pulse deficit 37. The systolic blood pressure measured $142 \mathrm{~mm}$. $\mathrm{Hg}$ and the diastolic 70 . The peripheral vessels were somewhat thickened. The lungs were clear on percussion but a few moist râles were heard at both 


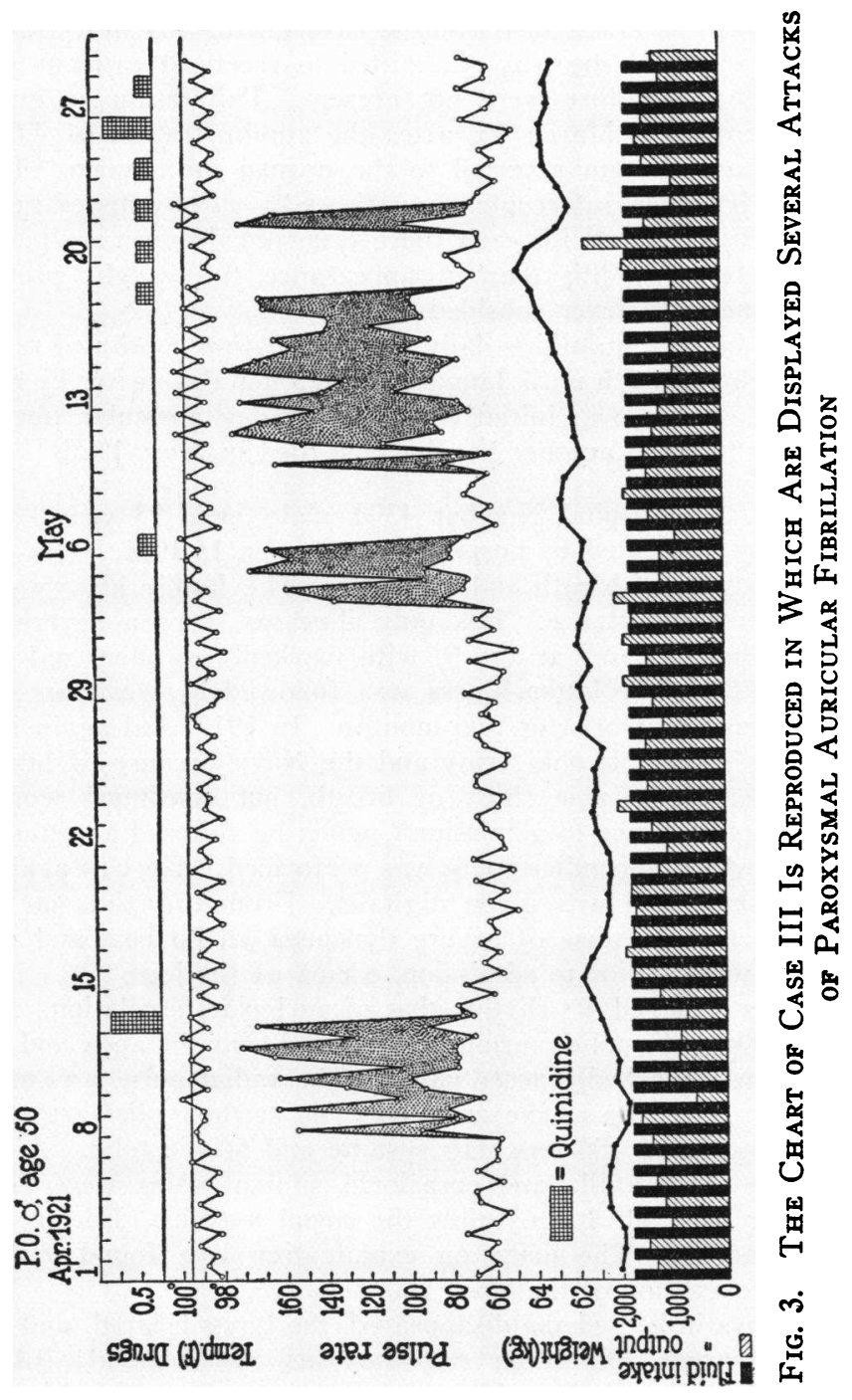


bases. The edge of the liver was felt $1 \mathrm{~cm}$. below the costal margin. The urine contained a faint trace of albumin. The Wassermann reaction of the blood was positive on several occasions.

For a year and a half the patient was observed in and out of the hospital. The auricles continued to fibrillate but by the judicious use of digitalis he was kept free of the symptoms of heart failure. He received also mercurial inunctions. His course was practically afebrile save during his first attack of mild failure. In March, 1921, he was readmitted to receive treatment with quinidine. Signs of heart failure were not present. Palpitation on exertion was the only complaint. On March 15, after the administration of 4.6 grams of quinidine, the heart rhythm reverted to the normal mechanism. The normal mechanism was, however, interrupted four times by paroxysms of rapid auricular fibrillation (Fig. 3). With each there occurred dyspnea, palpitation, gain in weight and fever. With their disappearance the weight promptly fell, palpitation, dyspnea and fever subsided.

Subsequently he took quinidine daily and the rhythm continued regular. He remained in excellent health until January 9,1923 , ten days after he had stopped taking quinidine on his own initiative. On this day auricular fibrillation recurred. He was taken to another hospital and died in May, 1923.

Case IV. T. S., Hospital Number 4390, male, aged 23 years, an elevator operator, was first admitted to hospital on October 1, 1921. His chief complaints were shortness of breath and sweating. The family history contributed no information of importance. His only illnesses were a severe attack of "inflammatory rheumatism" at age 9, with markedly swollen, red joints, and influenza at age 22 . The latter illness was followed by weakness which prevented his returning to work for two months. In 1917, and again in 1919, he was rejected for service in the Army and the Navy because of heart trouble. Shortly thereafter he became short of breath, but continued working until April 1921 (6 months prior to admission), when he suffered an attack of acute appendicitis for which appendicectomy was performed. He was again told that he had heart disease and was given digitalis. From this time on he was in bed most of the time because of severe dynspnea, orthopnea, and cough and, for four or five weeks prior to admission, edema of the legs.

His heart was enlarged, its rhythm that of auricular fibrillation. A blowing systolic and rumbling diastolic murmur were heard near the apex and the second pulmonic sound was markedly accentuated. The radial pulse was of poor and varying volume. The rate at the apex was 78 ; at the radial artery 70 . The blood pressure measured $100 \mathrm{~mm}$. Hg systolic and 60 diastolic. A few crepitant râles in the right axilla and occasional sibilant râles were heard. The edge of the liver was felt $1 \mathrm{~cm}$. below the costal margin. Edema was found only over the sacrum. The urine on examination was found to be normal. The Wassermann reaction of the blood was negative.

After four days in bed edema disappeared, the lungs cleared, and the degree of dyspnea decreased. Quinidine sulphate was administered; 0.4 gram on October 10, 1.2 gram on October 11, and 1.6 gram each on October 13 and 14. The heart rate increased to 140 , the temperature rose to $101^{\circ}$, severe palpitation and dyspnea appeared. On October 15 and again on October 16, digitan, 1.0 gram was administered. The heart rate now dropped promptly, the temperature fell, and the weight decreased. Quinidine was given again on October 24 and 25 , but once more tachycardia, gain in weight and fever followed, relieved by the exhibition of digitan on the following day. The use of quinidine had twice 


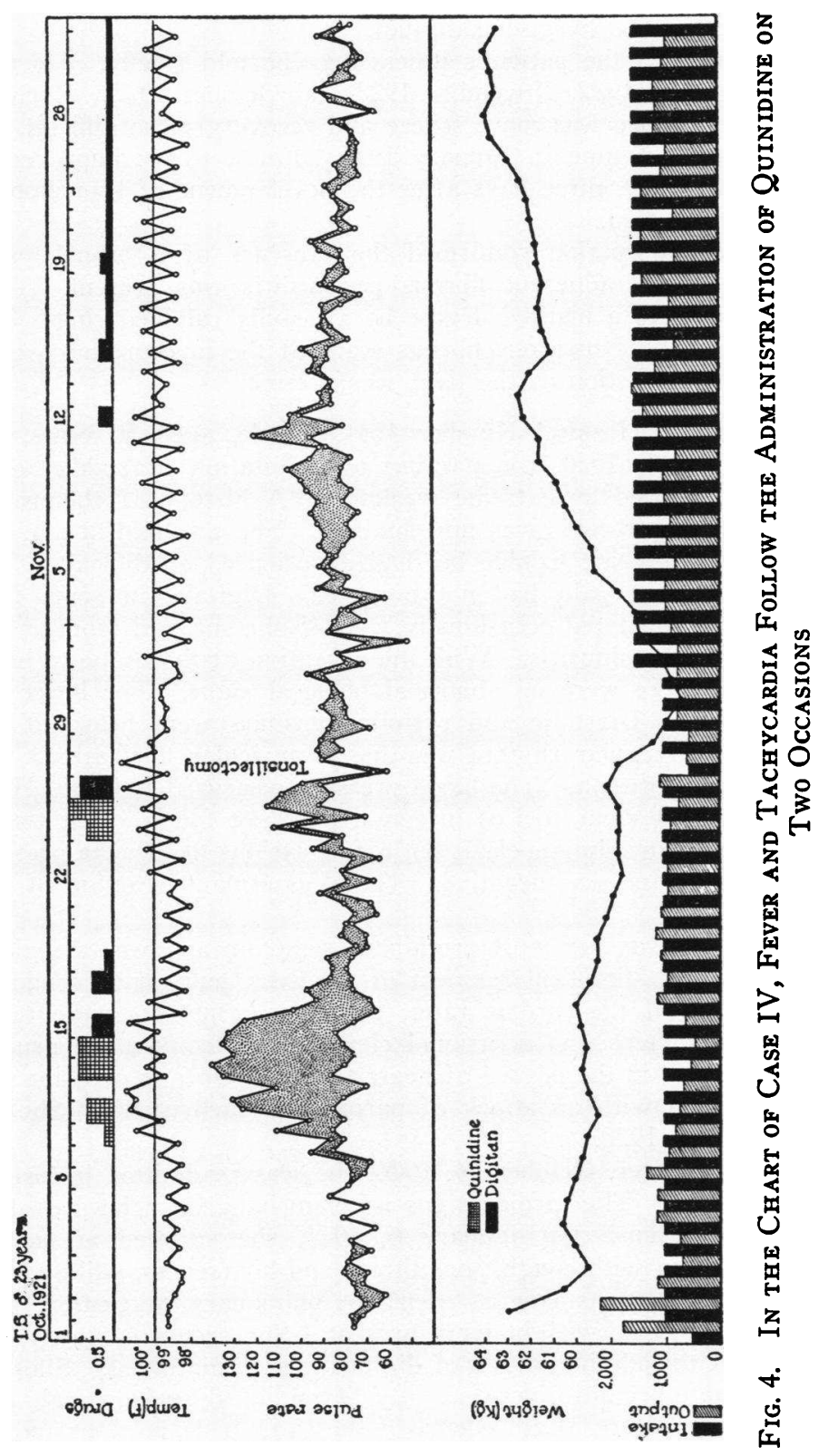


failed to restore the normal rhythm. Tonsillectomy was performed October 26 . Immediate recovery from the operation was uneventful, but the heart rate and the weight increased slowly. On November 11 fever appeared. All these symptoms were relieved by giving digitan.

The remainder of the patient's illness may be told briefly. He was readmitted in September 1922, November 1923 and October 1925. Each time the degree of heart failure was more severe and recovery more difficult. $\mathrm{He}$ was admitted for the last time in January 1926. For a while, improvement took place, but on March 9, three days after the development of bronchopneumonia with high fever, he died.

Postmortem examination confirmed the presence of a bronchopneumonia. The heart was large, adherent fibrous pericarditis was present. The mitral valve was stenosed to a marked degree by a heavily calcified ring. The other valves were normal. Numerous fibrous scars of the myocardium were found. Chronic passive congestion of the liver was present.

Case $V . R$. S., Hospital Number 4144, female, aged 50 years, was first admitted on April 1, 1920, complaining of palpitation of sudden onset nine weeks before, followed by extreme weakness. A history of rheumatic fever, chorea, sore throats or colds was not obtained. She was told at age 16 of the existence of serious heart trouble but had experienced no difficulty. Dyspnea, edema and precordial pain had not occurred. Digitalis in some form had, however, been administered occasionally. At 45 she suffered from an attack of influenza followed by sinusitis. With the exception of those due to her cardiovascular system there were no abnormal physical signs. The heart was considerably enlarged. Diastolic and systolic murmurs were heard at base and apex; the totally irregular rhythm was due to auricular fibrillation. No signs of congestion were present. The blood pressure measured $164 \mathrm{~mm} . \mathrm{Hg}$ systolic and 86 diastolic. Obvious foci of infection were not found. The tonsils were small and atrophic, the sinuses clear, but a few carious teeth were present. Examination of the urine was negative. The Wassermann reaction of the blood was negative.

At first, cough was her most prominent symptom and was associated with increase in dyspnea, slight enlargement of the liver, pulmonary congestion and moderate increase of the cardiac rate. All these symptoms were relieved by taking digitalis. There was occasional elevation of temperature usually associated with increase in the degree of heart failure. On one occasion it rose to $101^{\circ}$ in association with an attack of paroxysmal tachycardia. She was discharged August 21, 1920.

A few months later, October 24, 1920, she was readmitted in heart failure of moderate degree. The cardiac signs were unchanged. The use of digitalis relieved her symptoms. On January 6, 1921, she suffered an acute upper respiratory infection with cough, sore throat and hoarseness, while taking digitalis (Fig. 5a). She was free of edema or pulmonary congestion, but fever, tachycardia, and loss of weight were present. She recovered in 3 or 4 days. On February 26, the administration of digitan was discontinued. Shortly thereafter the heart rate began to increase. By March 17, dyspnea, cough and palpitation had become severe, slight edema of the shins had appeared and the liver had descended from $3 \mathrm{~cm}$. to $8 \mathrm{~cm}$. below the costal margin. Instead of loss in weight, a slight increase occurred. Fever was present until, with the administration of digitan on March 19, improvement began (Fig. 5b). During the latter part of April fever accompanying another acute upper respiratory infection occurred (Fig. 5c). At this time she was receiving digitan 0.1 gram a 


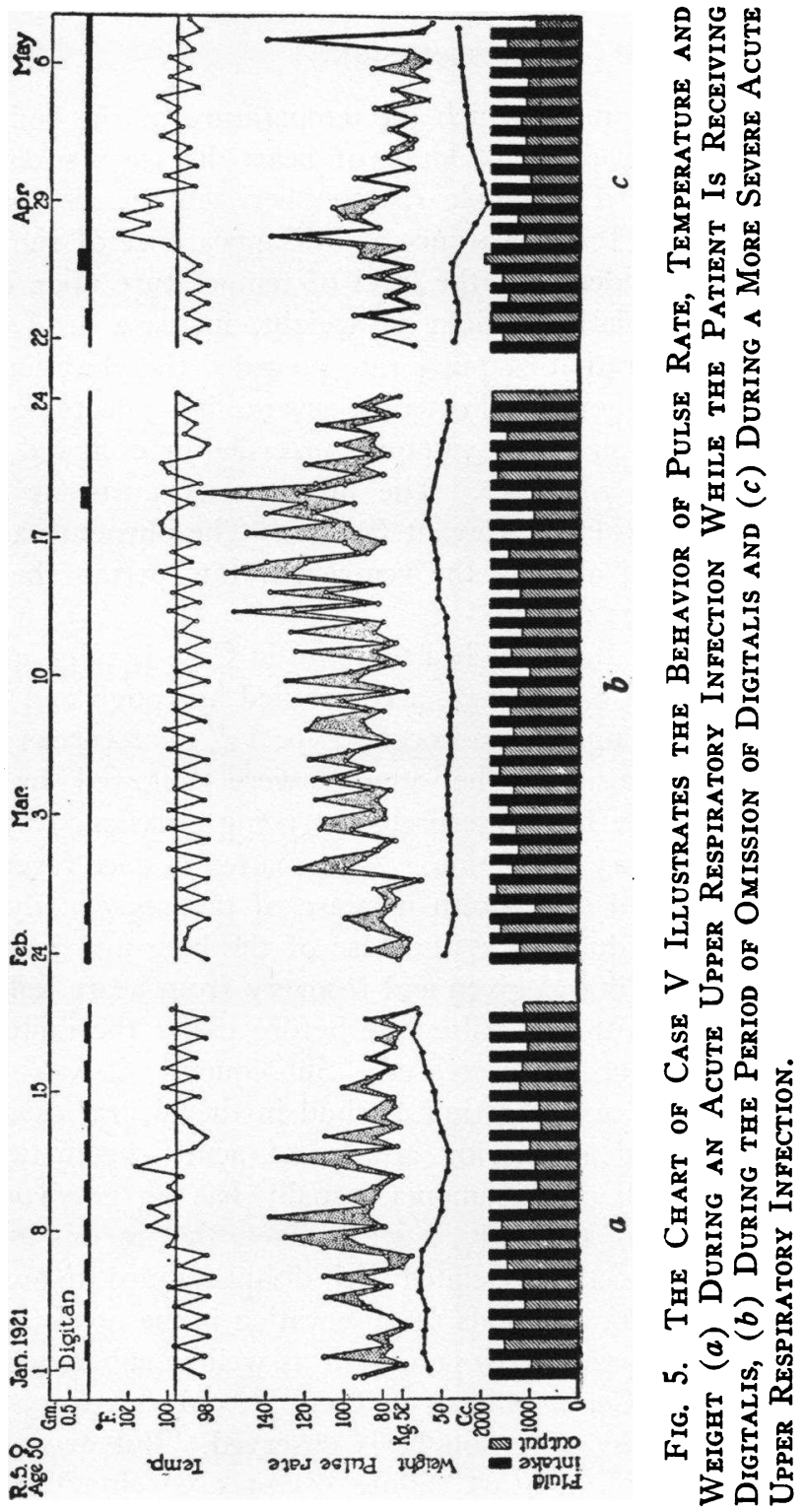


day. A loss in weight without noteworthy diuresis occurred but whether it was due to the administration of 0.3 gram digitan on the day before the rise in temperature is difficult or impossible to decide.

On June 24, 1921, she was discharged in a fair degree of compensation due to taking digitalis.

\section{DISCUSSION}

The circumstances under which the temperature of the body of these patients suffering from different kinds of heart disease rose and fell deserve close attention. The simple relation between the rise and fall of rectal temperature and the appearance and disappearance of signs of heart failure points to dependence of the level of temperature upon changes in the state of the circulation. Gain in weight, increase in dyspnea and palpitation, and acceleration of pulse rate preceded the elevation of rectal temperature by several hours and often by several days; decrease in weight, in pulse rate and in severity of symptoms preceded or occurred simultaneously with subsidence of fever. The presence of auricular fibrillation facilitated the analysis of the relevant factors. The phenomena which occurred as the result of altering the ventricular rate permit the view that fever and circulation are related.

During two of the attacks of heart failure in Case I, râles and dullness appeared at the bases of the lungs, accompanied by cough and expectoration of sputum containing Pneumococcus Type IV, micrococcus catarrhalis and other microorganisms. The attacks were followed by jaundice. These signs might have been interpreted as being occasioned by bronchopneumonia or pulmonary infarction. As a matter of fact fever appeared and gradually increased along with increase of tachycardia, dyspnea and gain in weight before dullness at the base of the lung and jaundice were noticed. When digitalis was given and recovery from heart failure began, the temperature fell with the pulse rate before either the dullness at the base of the lung or jaundice decreased. Subsequently it was shown that dullnss was due to an accumulation of fluid in the pleural cavity. Since giving digitalis to patients during attacks of acute rheumatic fever or during infections, such as pneumonia, usually has no noteworthy effect upon the temperature of the body, it seems likely that in this instance fall in temperature was directly associated with disappearance of heart failure.

One advantage of long periods of observation is the opportunity which is afforded of studying the development of, as well as subsequent recovery from, heart failure. Commonly, as in Case II, only recovery from that attack for which admission is sought is observed. But even in this instance, in which fever and heart failure disappeared simultaneously, the inference seems clear that interdependence of the two existed, for it was the success of treatment of the circulatory disturbance alone, in the course of which fever disappeared, that suggests dependence of the latter upon the former. Instances where the relation of fever to heart failure stand 
out more clearly are often to be observed in the occurrence of attacks of paroxysmal tachycardia. These attacks, especially if prolonged, may be associated with the progressive development of signs of heart failure; under these circumstances fever may be an accompanying phenomenon. Gradual gain in weight, increase in dyspnea and rise in rectal temperature which followed the onset of each of three paroxysms of rapid auricular fibrillation (Case III) is an illustration in point. The oniy discernible change in the physiological state of this individual was an abrupt change from the normal efficient cardiac rhythm to an abnormal and obviously very inefficient one. Further evidence of this relation is to be found in the abrupt, almost immediate return to normal temperature and the disappearance of dyspnea on the resumption of the normal rhythm (Fig. 3).

Another example is Case IV in which tachycardia, severe palpitation, dyspnea, gain in weight, and fever, followed on two occasions directly upon the administration of quinidine. All these symptoms disappeared promptly on taking digitalis (Fig. 4). It seems unlikely that exacerbation of a rheumatic process should twice coincide with giving quinidine. Unaccountable attacks of fever with acceleration of the pulse rate under these circumstances have been observed by others $(3,4)$. Pepper $(5)$ has noticed the development of leukocytosis and fever in cases of paroxysmal tachycardia. Fever obviously is not due directly to the administration of quinidine, for when there is no tachycardia there is no fever; on the contrary, when taking quinidine terminates an attack of auricular fibrillation, as in Case III, fever which has been present disappears. An instance (Case V), finally, has been chosen to illustrate the fact that it is possible in the same individual to distinguish between fever due to an infectious process and fever due to heart failure simply by the difference in behavior of the weight of the body alone-loss during infection (Fig. 5a and c); gain during heart failure (Fig. $5 \mathrm{~b}$ ).

Special effort was not made in these earlier experiences to secure evidence of hidden infection. Subsequently an attempt was made, however, to ascertain how frequently pulmonary infection was demonstrable in connection with these phenomena. To secure material for culture, the lungs were punctured in thirty-four patients, all but six the subject of fever. Forty punctures were made. Only when frothy, mucoid material was drawn into the syringe-tested for tightness in the subcutaneous tissuewere the cultures regarded as having been made of material obtained from the lungs. That portion of the lungs which cast the densest shadows in $\mathbf{x}$-ray photographs, found invariably near one base, was selected for exploration. Growth on ordinary aerobic media failed to occur in 36 instances. There were four in which growth occurred; two were contaminated (B. subtilis); in one, Streptococcus viridans was recovered (several cultures from this patient's blood were negative) and in a fourth Bacteriodes acuminatus, a terminal invader, found also in postmortem cultures the following day. 
Nine postmortem examinations were performed. Blood cultures made in all the patients before death were negative. In five instances pulmonary infarcts were found. These were sterile except one from which $B$. coli and a gram-positive bacillus were cultivated. Direct cultures of pulmonary tissue were made. In seven of the nine cases material obtained both antemortem and postmortem was sterile. In the eighth case Bacteriodes acuminatus obtained before death by lung puncture, was obtained postmortem also from the lungs, the heart's blood and the pleural fluid. In the ninth case postmortem one lung contained Staphylococcus hemolyticus and the other was sterile. Material obtained before death by puncture of the lungs was sterile. From these experiences there appears no reason for regarding the lungs as infected.

These studies recall Thacher's (6) observations concerning fever in heart disease. In 1905 he commented on autopsies of a large number (901) of cases of chronic endocarditis, many (505) of which exhibited fever though its cause in more than half (291) was obscure. Exacerbation of the rheumatic process would doubtless be assigned now as the most likely explanation of its occurrence. But Thacher felt forced to remark somewhat forlornly that "the autopsy as well as the clinical picture awarded nothing which would have been apt to produce fever unless simple passive congestion of the viscera can do so."

Clearly, however extensive the search for an infectious source, failure to find it does not assure its absence. On the other hand that the train of events itself in heart failure may lead to the occurrence of fever is an idea which has occasionally been entertained, as the quotation from Thacher shows. In fact von Bärensprung (7) (1852) ventured to suggest that fever accompanied hypertrophy of both ventricles, or particularly that of the left when the volume of the pulse was great, while subnormal temperatures occurred when the pulse was small and the right ventricle dilated.

There is evidence, furthermore, of a different nature that fever may be circulatory in origin. Following death, when the circulation has ceased the temperature of the body sometimes continues to rise. Wunderlich (1) (p. 287) calls attention to Seume's (8) first having observed (1856) this phenomenon and to his own confirmation of this observation. In studying further the mechanism underlying this idea Heidenhain (9) demonstrated (1870) in dogs that stopping the respiration resulted in a fall of temperature while cessation of the heart beat brought about a rise and he noticed also that compression of the thoracic aorta was followed by a rise in temperature of the liver and intestinal contents. A few years later Ackermann (10) showed, also in dogs, that rise of the internal temperature immediately after death took place while the peripheral temperature was continually falling. Since the blood pressure was zero, and since artificial respiration was still in progress the rise in rectal temperature could 
be attributed only to cessation of the circulation. Based likewise on the idea that level of temperature is modified by conveyance away by the blood stream of heat locally developed, Binger and Christie (11) have more recently shown that in lungs in which the circulation is stopped local temperature rises promptly with the introduction of artificial heat (diathermy). Nothing has been found in the literature to indicate, however, to what degree this phenomenon occurs when the circulation is slowed rather than stopped. In a similar sense, also with regard to the transportation of heat to the periphery, elevation of the temperature occurs in "sympathetic fever" (12), after the administration of cocaine (13) and after puncture of the corpus striatum (14), due apparently to diverting the circulation of blood in large measure from the surface of the body.

In heart failure when the circulation as a whole, to the skin as well as to other parts of the body, is slowed, it seems likely, accordingly, that elevation of the temperature in the interior parts of the body may occur. But it is quite possible that the sequence of events leading to the accumulation of heat is not so direct: the so-called heat centers of the brain may be incapacitated through faulty circulation and may fail to regulate, through faulty vasomotor arrangements at the surface of the body, the loss of heat from the skin. Another possible origin of fever-chemical in nature-is the formation in the tissues, due to lack of oxygen, of a substance toxic in nature and capable of inducing fever. Such substances have been found by Krehl and Matthes (15) and many others during the course of infectious diseases. Mandel (16) has in fact demonstrated the presence of such a substance in the urine of patients suffering with aseptic (surgical) fevers.

A discussion on the ultimate mechanism involved in the causation of non-infectious fever is, however, beyond the scope of the present study. At the moment no more is intended than to describe a relation between heart failure and fever which in certain instances suggests dependence of the latter (fever) upon some process involved in the former (heartfailure).

\section{SUMMARY}

1. Fever may occur during heart failure in the absence of evidence of infection or of the non-infectious conditions which have been enumerated and which are likewise associated with the development of fever.

2. Results of bacteriologic studies of material obtained by puncture of the lungs during life and from the lungs at autopsy in patients with heart failure accompanied by fever are presented.

3. In a number of cases signs of heart failure appear or begin to increase just prior to the occurrence of fever; fever and the signs of heart failure disappear simultaneously. 
4. These relations suggest that the occurrence of fever in these instances is dependent upon the mechanisms involved in heart failure itself.

\section{BIBLIOGRAPHY}

1. Wunderlich, C. A., On the temperature in diseases. A manual of medical thermometry. New Sydenham Society, London, 1871. Translated from the 2d German ed. by W. B. Woodman.

2. Romberg, E., Lehrbuch der Krankheiten des Herzens und der Blutgefässe. 3 Aufl. Stuttgart, 1921, p. 200.

3. Winternitz, M., Zur Chinidintherapie des Vorhofflimmerns. Med. Klin., $1932,28,1308$.

4. Bourne, G., Three cases of paroxysmal auricular flutter. Lancet, 1933, 1, 686.

5. Pepper, O. H. P., Non-infectious leukocytosis. M. Clin. North America, 1924, 8, 717.

6. Thacher, J. S., Fever in chronic endocarditis. Trans. Assn. Amer. Phys., 1905, 20, 70.

7. v. Bärensprung, F., Untersuchungen über die Temperaturverhältnisse des Menschen im gesunden und kranken Zustande. Arch. f. Anat., Physiol., u. wissensch. Med., Berlin, 1852, 217.

8. Seume. Quoted by Wunderlich, page 287. "de Calore corporis humani in Morte observato" 1856.

9. Heidenhain, R., Ueber bisher unbeachtete Einwirkungen des Nervensystems auf die Körpertemperatur und den Kreislauf. Arch. f. d. ges. Physiol., $1870,3,504$.

10. Ackermann, Th., Ueber die physiologischen Wirkungen des Digitalins auf den Kreislauf und die Temperatur. Deutsches Arch. f. klin. Med., 1873, $2,125$.

11. Binger, C. A. L., and Christie, R. V., An experimental study of diathermy. I. The measurement of lung temperature. J. Exper. Med., 1927, 46, 571.

12. Cramer, W., Fever, heat regulation, climate, and the thyroid-adrenal apparatus. Longmans, Green and Co., London, 1928.

13. Marshall, H. T., Aydelotte, B. F., and Barbour, H. G., Heat regulation and water exchange. XIII. Insensible weight loss and liver hydration in the onset of cocaine fever. Am. J. Physiol., 1931, 98, 615.

14. Aronsohn, Ed., und Sachs, J., Die Beziehungen des Gehirns zur Körperwärme und zum Fieber. Arch. f. d. ges. Physiol., 1885, 37, 232.

15. Krehl, L., und Matthes, M., Wie entsteht die Temperatursteigerung des fiebernden Organismus? Arch. f. exper. Path. u. Pharmakol., 1897, 38, 284.

16. Mandel, A. R., The alloxuric bases in aseptic fevers. Am. J. Physiol., 1904, 10, 452. 